\title{
Energy and Particle Confinement in MAST
}

\author{
M. Valovič, H. Meyer, R. Akers, C. Brickley, N. J. Conway, G. Cunningham, A. Kirk, \\ B. Lloyd, D. Taylor, M. J. Walsh1) and the MAST Team
}

EURATOM/UKAEA Fusion Association, Culham Science Centre, Abingdon, Oxon OX14 3DB, UK

1)Walsh Scientific Ltd Culham Science Centre, Abingdon, Oxon OX14 3EB, UK

e-mail contact of main author: martin.valovic@ukaea.org.uk

\begin{abstract}
Quasi-stationary H-mode plasmas have been produced on MAST and submitted to the ITPA H-mode confinement database. The MAST data are expanding the database by a factor of 2.2 in inverse aspect ratio $\varepsilon=$ $a / R$ and by a factor of 2.5 in toroidal $\beta$. The MAST energy confinement times broadly agree with the IPB98(y,2) scaling law. Merging with the published international database shows that the energy confinement time in MAST supports an $\varepsilon$ dependence consistent with the subset of conventional shaped cross-section tokamaks when represented in engineering parameters. In dimensionless parameters, MAST indicates a collisionality dependence stronger than in the IPB98(y,2) scaling. MAST data strongly link $\varepsilon$ and $\beta$ scalings and as a result the proposed $\beta$ independence would imply weak $\varepsilon$ dependence. L-mode confinement exceeds the value given by the current Lmode scaling suggesting a stronger $\varepsilon$ dependence than predicted. In $\mathrm{H}$-mode, the particle transport in the core is dominated by the Ware pinch and the neutral beam source.
\end{abstract}

\section{Introduction}

Fusion physics and plasma engineering is presently focusing on the preparation of ITER operation, a machine with medium aspect ratio geometry. In parallel, further evaluation of confinement formulas for all toroidal devices continues to be a major thrust of magnetic fusion research. The data from the low aspect ratio tokamak MAST are significantly extending International Tokamak Databases and imposing powerful constraints on dependencies of transport on dimensionless parameters. In addition, MAST data are helping to remove degeneracy related to the aspect ratio and thus improving the predictive capability of scalings towards ITER and next step spherical tokamaks. This paper reports on dedicated experiments on MAST aimed to extract data for input to International Databases. The data are discussed in the context of the published H-mode confinement dataset.

\section{ELMy H-mode dataset from MAST}

Quasi-stationary H-mode plasmas have been produced on MAST in order to provide the data for heat and particle transport at low aspect ratio. The data are selected to satisfy the conditions for the ITPA database. In particular the aim is to establish plasmas that are quasistationary for the maximum possible period to reduce the uncertainties due to the time derivatives. The review sheet of a typical discharge is shown in figure 1 . The time of data extraction is selected so that the rate of change of energy content is smaller than $15 \%$ of the power loss and is close to the measurement time of high resolution Thomson scattering and charge exchange diagnostics. 
The dataset covers the engineering parameter range of plasma current $I_{p}=(0.73-0.78) M A$, geometric major radius $R_{\text {geo }}=(0.8-0.83) \mathrm{m}$, minor radius $a=(0.54-0.57) \mathrm{m}$, elongation $\kappa=1.9-2.0$, vacuum toroidal field at geometric radius $B=(0.45-0.49) T$, line averaged density $\bar{n}_{e}=(3.0-5.4) \times 10^{19} \mathrm{~m}^{-3}$ and thermal heat power loss $P_{L, t h}=(1.5-3.0) \mathrm{MW}$. All data are with double null divertor configuration. The working gas is deuterium and plasmas are heated with neutral beams. Typically one of two beam lines is operated in hydrogen to improve the ion temperature measurement but nevertheless the neutral particle analyser shows that the effective mass is $M_{\text {eff }}=1.94-2.0$. The measured effective charge at mid radius is $Z_{\text {eff }}=1.1-1.4$.

The thermal energy confinement time has been deduced as $\tau_{E}=W_{t h} / P_{L, t h}$, where $W_{t h}=$ $W_{M H D}-W_{F}$ and $P_{L, t h}=P_{o h}+P_{N B}-d W_{M H D} / d t$. Here, $W_{M H D}$ is the energy content determined from EFIT equilibrium reconstruction. Fast ion energy content $W_{F}$ and absorbed beam power $P_{N B}$ are deduced from TRANSP analysis [1]. The contribution of rotation to the energy content is small $(\sim 7 \%)$. The power loss due to shine through, charge exchange and orbit loss is several percent in total. The total radiated power is below $15 \%$ and is not allowed for when calculating included in the confinement time in line with the convention of the international database. The energy content $W_{M H D}$ from magnetic reconstruction and that calculated by TRANSP using kinetic profiles agree within $\pm 10 \%$.

It is found that the fast ion content increases as plasma collisionality decreases as seen in figure 2. In order to limit the uncertainty in thermal energy confinement and also exclude the possible effect of fast particles on heat transport we have limited data to $W_{F} / W_{M H D}<35 \%$. This basically defines the lower limit on density range in the dataset as specified above.
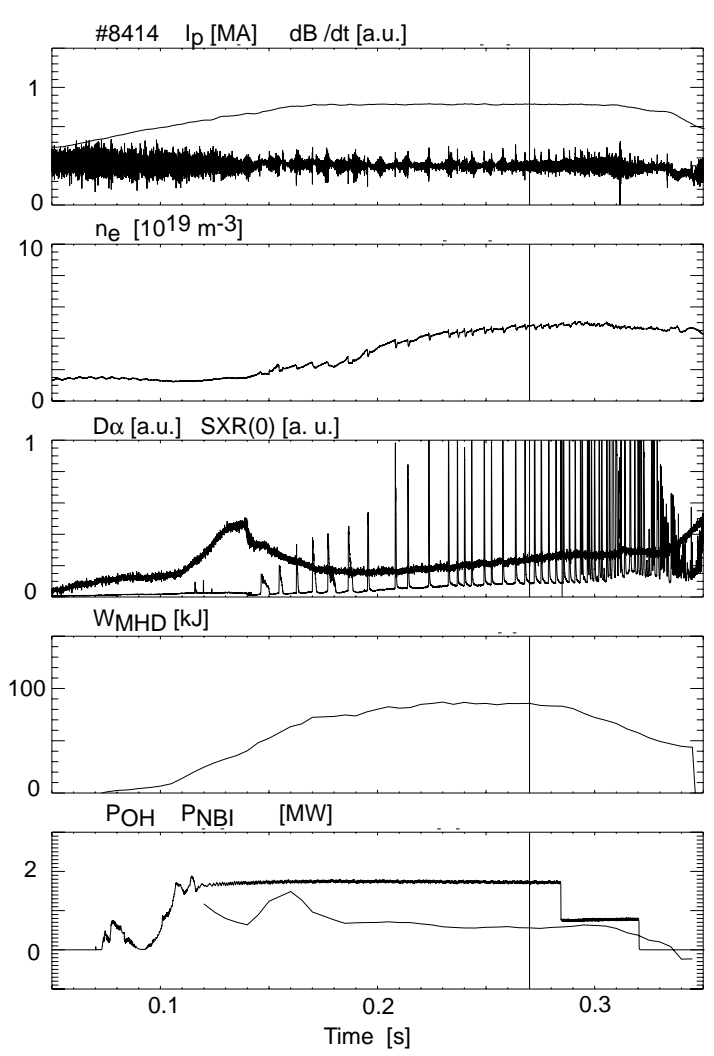

FIG.1. Review sheet of typical plasma used for confinement studies. The vertical line shows the time of extraction of the data.

$\mathrm{W}_{\mathrm{F}} / \mathrm{W}_{\mathrm{MHD}}(\%)$

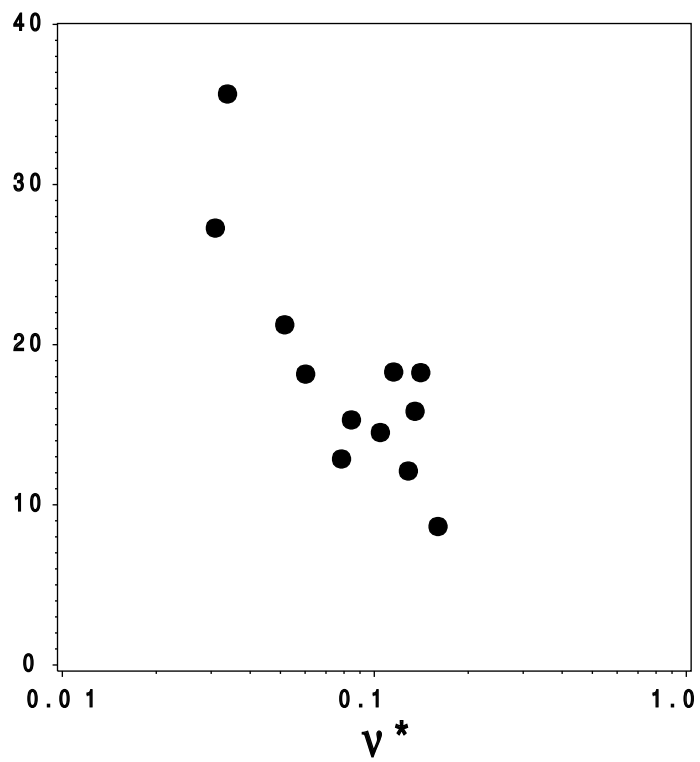

FIG.2. Dependence of fraction of fast particle energy content on normalised collisionality. 


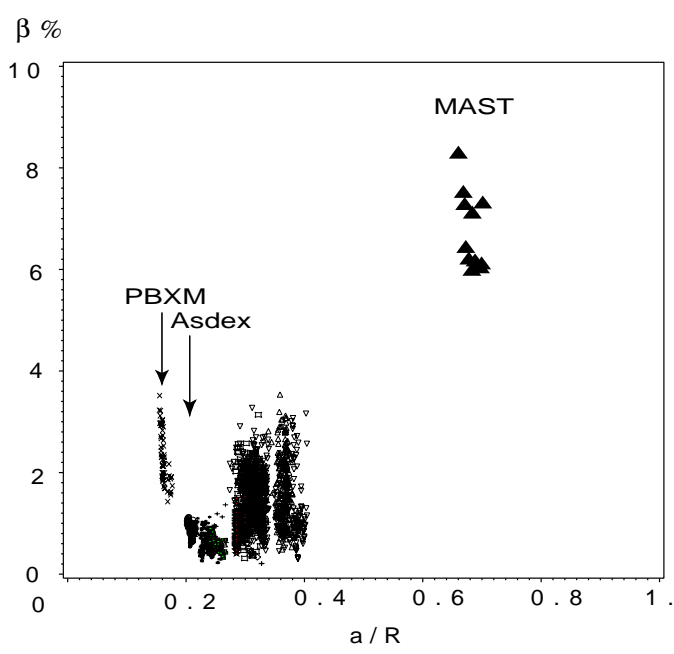

FIG.3. MAST data merged with public version of International H-mode confinement database DB03V5.

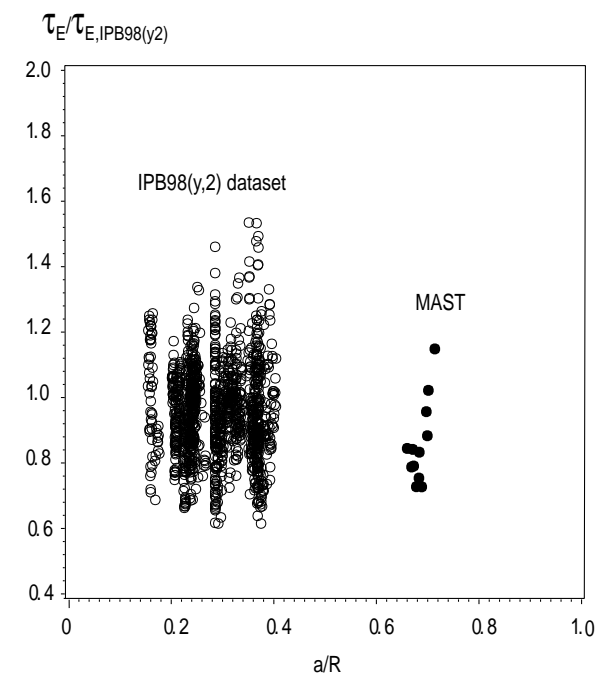

FIG.4. Energy confinement time normalised to IPB98(y,2) scaling plotted against inverse aspect ratio.

The dataset has been formatted and submitted to the International H-mode confinement database and has been incorporated in recent scalings. For this paper, we merged the MAST data with the older version of the international H-mode confinement database DB03V5 that has been released to the public. In particular we used the subset from which the confinement scaling IPB98 $(y, 2)$ has been derived [2]. Figure 3 shows this combined dataset. It is seen that the MAST data are expanding the range of inverse aspect ratio from $\varepsilon=0.15-0.40$ to $\varepsilon=0.15-0.71$ i.e. by a factor of 2.2. Adding the MAST data makes the combined dataset more symmetric along the $\varepsilon$ axis with respect to the ITER point $\varepsilon_{\text {ITER }}=0.32$. MAST data are also improving the conditioning of the dataset so that now the spread in $\varepsilon$ is to a large extent given by tokamaks with conventional shaped plasma cross-sections in comparison with the previous situation when the $\varepsilon$ range was dominated by data from the bean-shaped PBXM and circular plasmas in Asdex. Simultaneously, as seen from figure 3, MAST is extending the range of thermal toroidal beta by a factor of 2.5 from $\beta \%=0.22-3.5$ to $\beta \%=0.22-8.5$. Concerning the other parameters such as normalised Larmor radius $\rho^{*}$, normalised collisionality $v^{*}$, safety factor $q$ and elongation $\kappa$, the MAST data fall inside the range defined by the IPBH98 $(\mathrm{y}, 2)$ dataset, although for $\rho^{*}$ and $\kappa$ MAST data are just at the upper end of the ranges. Conventionally the dimensionless parameters are defined using the volume averaged temperature $T \propto W_{t h} / \bar{n}_{e} V$.

\section{Engineering parameters confinement scaling}

The largest effect of MAST data is expected on the aspect ratio dependence of the confinement scaling. Figure 4 shows the energy confinement time normalised to the IPB98 $(y, 2)$ scaling plotted against $\varepsilon$. It is seen that the MAST data agree with the scaling in the sense that the spread of residuals $H_{98 y 2}=\tau_{E} / \tau_{I P B 98(y, 2)}=0.7-1.15$ for MAST is even 
somewhat smaller than the range of residuals of the IPB98 $(y, 2)$ dataset $H_{98 y 2}=0.6-1.5$. This means that in the first approximation MAST data do not change the scaling.

In the IPB98(y,2) scaling, the closed divertor correction TAUC92 and area-defined elongation $\kappa_{a}$ were introduced in order to accommodate the PBXM, Asdex and PDX tokamaks [2, 3]. However, these devices have large leverage on the $\varepsilon$ dependence as seen in figure 3 and these corrections could, in principle, affect the aspect ratio dependence in the scaling. To test whether MAST supports the $\varepsilon$ dependence of the IPB98(y,2) scaling the aforementioned tokamaks have been removed from the dataset and the MAST data added. Log-linear regression in engineering parameters, $\tau_{E} \propto I^{\alpha l} B^{\alpha B} \bar{n}_{e}^{\alpha n} R^{\alpha R} M^{\alpha M} \varepsilon^{\alpha \varepsilon} \kappa_{a}^{\alpha \kappa}$, is used together with the Kadomtsev constraint just as for the IPB98(y,2) scaling. The results of this analysis are summarised in table $\mathrm{I}$. It is seen that the inverse aspect ratio exponent $\alpha_{\varepsilon}$ remains approximately the same as in the IPB98(y,2) scaling with somewhat stronger dependence given by MAST. This fact does not change if MAST data are weighted 10 times more strongly as seen in the last row of table I. These results show that to a first order approximation the present MAST data support the recommended H-mode scaling law.

TABLE I: EFFECT OF MAST DATA ON POWER LAW SCALING.

\begin{tabular}{|l|c|c|c|c|c|c|}
\hline Dataset & $\alpha_{\varepsilon}$ & $x_{\rho *}$ & $x_{\beta}$ & $x_{v^{*}}$ & $x_{\varepsilon}$ & RMSE(\%) \\
\hline IPB98(y,2) & 0.58 & -2.67 & -0.92 & -0.01 & 0.73 & 14.5 \\
\hline IPB98(y,2) - (PBXM, PDX, Asdex)+MAST & 0.81 & -3.0 & -0.80 & 0.00 & 0.83 & 13.8 \\
\hline Above with weight(MAST) $=10$ & 0.80 & -3.0 & -0.84 & -0.01 & 0.74 & 14.4 \\
\hline
\end{tabular}

\section{Dimensionless parameters scalings}

To understand the real range of extrapolation to next step devices, the confinement scalings have to be linked to plasma physics variables. As a first step, the energy confinement time is expressed as a function of the normalised Larmor radius $\rho^{*}$, toroidal beta $\beta$ and collisionality $v^{*}$ in the form $B \tau_{E} \propto \rho_{*}^{x \rho^{*}} \beta^{x \beta} \nu_{*}^{x \nu^{*}} M^{x M} q^{x q} \mathcal{E}^{x \varepsilon} \kappa_{a}^{x \kappa}$. The exponents of the dimensionless parameters are derived by algebraic transformation from the exponents of the engineering variables similarly as in [2]. Table I shows the exponents of the dimensionless parameters derived by such a method. It is seen that the MAST data are not significantly changing the dependencies on dimensionless parameters when this methodology is applied. The scaling remains Gyro-Bohm $\left(x_{\rho^{*}} \approx-3\right)$ and the $\beta$ degradation and $v^{*}$ independence, as observed in the IPB98(y,2) scaling, are retained. In addition, the relatively strong dimensionless aspect ratio dependence (exponent $x_{\varepsilon}$ ) is unchanged.

\section{1. $v^{*}$ scaling}

The largest extrapolation from present MAST data to possible next step spherical tokamak devices such as a compact Component Test Facility (CTF) [4] is in the normalised collisionality, $\min \left(v_{*}^{\text {MAST }}\right) / \nu_{*}^{C T F}=90$. Extrapolation in normalised beta is small, $\max \left(\beta_{N}^{\text {MAST }}\right) / \beta_{N}^{C T F}=0.75$, although the extrapolation in toroidal $\beta$ is twice larger due to the higher $q$ in MAST. Interestingly values of $\rho^{*}$ are already covered $\max \left(\rho_{*}^{\text {MAST }}\right) / \rho_{*}^{\text {CTF }}=0.9$. 
Therefore the prediction of confinement in a CTF is very sensitive to the $v^{*}$ exponent in the power law scaling.

Figure 5 shows that plasmas with lower collisionality have better normalized confinement than high collisionality plasmas. This trend echoes that in DIII-D and ASDEX Upgrade data. Correlation of $v^{*}$ with $\rho^{*}$ and $\beta$ is relatively small so that this dependence can be attributed to physical effects of collisionality. Such misalignment of data and the scaling seems to be related to the well known discrepancy between $v^{*}$ independence in the $\operatorname{IPB} 98(\mathrm{y}, 2)$ scaling and a weak favourable $v^{*}$ dependence found in dedicated scans [5]. It is thought that this difference arises from the correlated errors in the

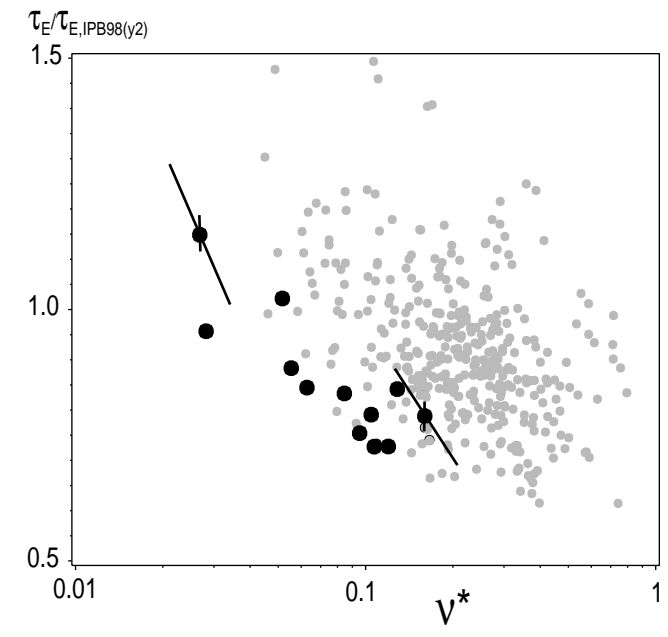

FIG.5. $\quad H_{98 y 2}$ plotted against $v^{*}$ for MAST. The error ellipses correspond to \pm 10 and $\pm 12 \%$ errors on $P_{\text {Lth }}$ and $W_{\text {th }}$ resp. DIII-D and ASDEX Upgrade data are shown in grey. database [3]. For MAST data this is illustrated in figure 5 which shows that the directions of the major axes of the error ellipses are very close to the direction of the data trend. Therefore part of the dependence could be simply due to the data scatter and conventional regression using $v^{*}$ as an independent variable is inapplicable, as it would overestimate the trend. For MAST data in figure 5 such regression gives $H_{98 y 2} \propto \nu_{*}^{-0.23}$ that could be interpreted as an upper estimate for the actual dependence.

The uncertainty in dimensionless exponents has been assessed previously using the width of RMSE minima [6]. For a somewhat larger subset of the DB03V5 dataset than used for the IPB98 $(y, 2)$ scaling, this analysis gave the range of exponents $B \tau_{E} \propto \nu_{*}^{-0.10 \pm 0.08}$. Recently an "error-in-variable" technique [7, 8] led to a somewhat larger uncertainty $B \tau_{E} \propto \nu_{*}^{-0.18 \pm 0.13}$.

\section{2. $\varepsilon-\beta$ interplay}

MAST data make a strong link between the $\varepsilon$ and $\beta$ dependences in the scaling because they expand the database by a factor of two simultaneously in both parameters. At the same time MAST data are roughly in agreement with the dependence $\tau_{E} \sim \mathcal{E} / \beta$ as seen in table I. Therefore if the scaling is constrained to have a more positive $\beta$ exponent in order to align with dedicated dimensionless scans in single machines $[7,8]$ then this should be compensated by a negative shift of the $\varepsilon$ exponent.

To quantify the scale of this interplay the set of dimensionless variables was reduced to $\rho^{*}$, $\beta, v^{*}$ and $\varepsilon$ by selecting the data from a narrow range of safety factor, elongation and mass: $q_{\text {eng }}=2.1-2.5, \kappa_{a}=1.59-1.8, M_{\text {eff }}=1.94-2$. Such a dataset contains $N=111$ data points and includes all MAST data plus selected data from JET, DIII-D and ASDEX Upgrade. Assuming gyro-Bohm scaling and $\beta$ degradation the log-linear regression gives 
$B \tau_{E} /\left(\rho_{*}^{-3} \beta^{-1}\right) \propto \varepsilon^{0.77}$ in line with the IPB98(y,2) scaling as given in Table I. Because $\rho^{*}$ and $\beta$ depend on the aspect ratio as well, the regression was performed with $\varepsilon$ removed from the definitions of these dimensionless variables. After the fit the $\varepsilon$ exponent was then recalculated in accordance with the original definitions of $\rho^{*}$ and $\beta$. When the scaling is constrained to be $\beta$ independent then regression gives a more negative exponent $B \tau_{E} /\left(\rho_{*}^{-3} \beta^{0}\right) \propto \mathcal{E}^{-0.58}$ as expected from the interaction between $\varepsilon$ and $\beta$ exponents due to the MAST data. Introducing the collisionality dependence up to $\tau_{E} \propto \nu_{*}^{-1 / 3}$ or considering weaker than gyro-Bohm scaling $\tau_{E} \propto \rho_{*}^{-2.7}$ results in the $\varepsilon$ exponent becoming more negative still. Further narrowing of the database to include only similar size tokamaks DIII-D and ASDEX Upgrade and MAST results in a database of $N=40$ data points and gives a weaker aspect ratio dependence $B \tau_{E} /\left(\rho_{*}^{-3} \beta^{0}\right) \propto \varepsilon^{-0.19}$.

The range of $\varepsilon$ exponents described above is consistent with a previous study [6] using RMSE minima that resulted in a weaker $\beta$ degradation and an $\varepsilon$ exponent of $B \tau_{E} /\left(\rho_{*}^{-2.8} \beta^{-0.5} \nu_{*}^{-0.1}\right) \propto \varepsilon^{-0.5 \pm 0.5}$. The above analysis also shows that the recently discovered interaction between $\beta$ and $v^{*}$ exponents in dimensionless scaling [7, 8] should be extended to 3 variables and include aspect ratio as well.

\section{L-mode engineering parameters scaling}

The recommended thermal energy confinement scaling for L-mode is almost $\varepsilon$ independent [2]. This is in contrast with the H-mode scaling and thus the L-H confinement enhancement

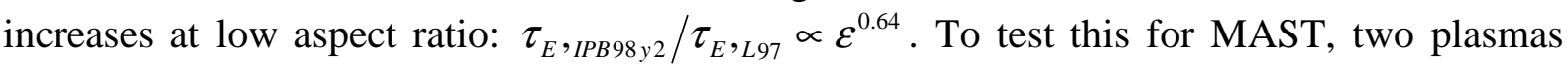
have been prepared, one in H-mode and one in L-mode having similar engineering parameters as seen in table II. For the L-mode plasma the L-H transition is inhibited by a small vertical displacement. For these conditions the scaling laws predict significant difference in L- and $\mathrm{H}$ mode confinement $\tau_{E}{ }_{I P B 98 y 2} / \tau_{E},{ }_{L 97} \approx 1.9$. The measured confinement times show, however, a much smaller factor $\tau_{E},{ }_{H} / \tau_{E},{ }_{L} \approx 1.4$. As seen in table II this is mainly because the L97 scaling underestimates the energy confinement in L-mode. These results may suggest that Lmode scaling should be $\varepsilon$ dependent similar to its H-mode counterpart.

Most of the difference between H-mode and L-mode global confinement is due to the lack of the pedestal in L-mode as indicated in table II by the electron energy content calculated from high resolution Thomson scattering data. This is also accompanied by more peaked profiles in L-mode with the electron pressure peaking factor of $p_{e}(0) /\left\langle p_{e}\right\rangle_{v o l} \approx 2.7$. Interestingly this relatively high value is close to the value assumed in a compact CTF device [4].

TABLE II: COMPARISON OF L-MODE AND H-MODE CONFINEMENT

$I_{p}=0.78 M A, R_{\text {geo }}=0.80 \mathrm{~m}, a=0.56 \mathrm{~m}, \kappa=1.9-2.0, B_{T}=0.49 T, M_{\text {eff }}=1.94$

\begin{tabular}{|l|c|c|c|c|c|c|c|}
\hline & $\begin{array}{c}\bar{n}_{e} \\
{\left[10^{19} m^{-3}\right]}\end{array}$ & $\begin{array}{c}P_{L, t h} \\
{[M W]}\end{array}$ & $\begin{array}{l}\tau_{E} \\
{[m s]}\end{array}$ & $\begin{array}{c}\tau_{E, L 97} \\
{[m s]}\end{array}$ & $\begin{array}{c}\tau_{E, I P B 98 y 2} \\
{[m s]}\end{array}$ & $\begin{array}{c}W_{e, p e d} \\
{[\mathrm{~kJ}]}\end{array}$ & $\begin{array}{c}W_{e} \\
{[\mathrm{~kJ}]}\end{array}$ \\
\hline $\mathrm{L} \mathrm{\# 8505}$ & 3.9 & 2.4 & $24 \pm 4$ & 18 & 34 & 2 & 38 \\
\hline $\mathrm{H} \# 8503$ & 4.6 & 2.4 & $34 \pm 5$ & 21 & 39 & 11 & 45 \\
\hline
\end{tabular}



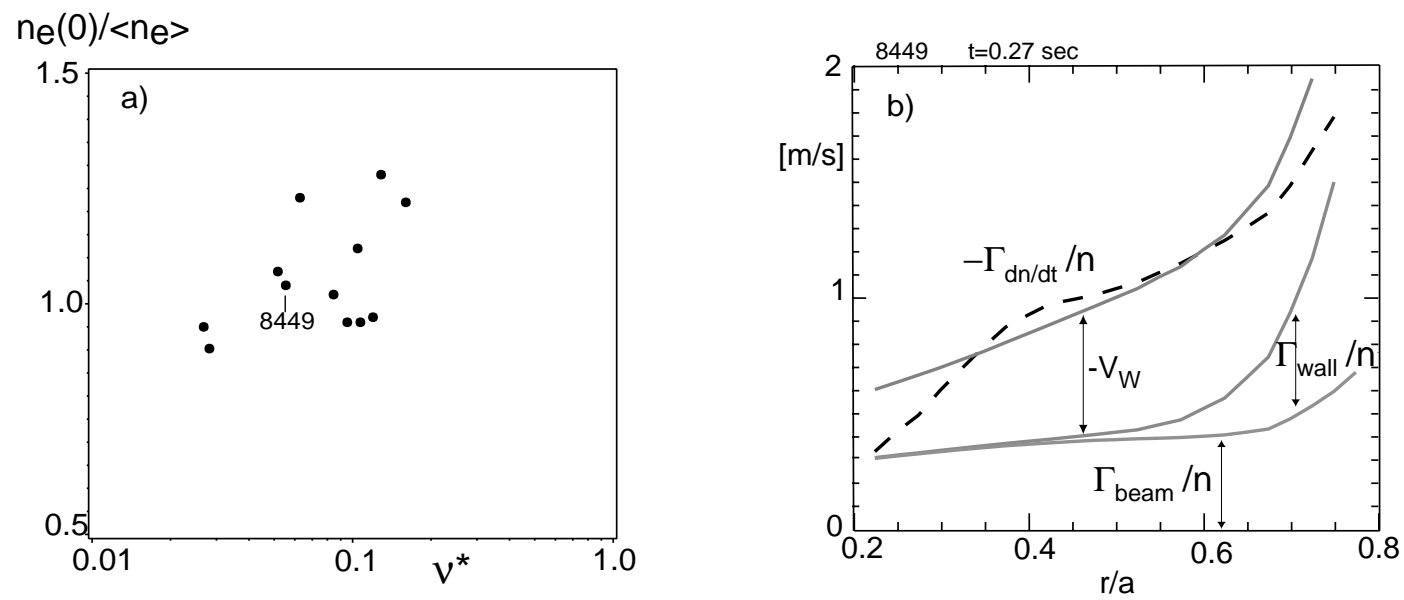

FIG. 6. a) The ratio of central to volume averaged density, against collisionality. Density peaking is taken from high spatial resolution Thomson scattering measurements. b) Particle balance as calculated by TRANSP. $\Gamma_{d n / d t}=-d / d t\left(\int n_{e} d V\right) / \int d S$ (dotted line).

\section{Particle balance}

In addition to energy confinement, the performance in future devices is sensitive to the shape of the density profile. In particular, peaked density profiles are favoured for devices such as a CTF for effective off axis current drive. In MAST, rather peaked density profiles are observed in L-mode with the ratio of central to volume averaged density of $n_{e}(0) /\left\langle n_{e}\right\rangle_{v o l}=1.5$ as found for shot \#8505 in table II. For H-mode, the density profiles are relatively flat with $n_{e}(0) /\left\langle n_{e}\right\rangle_{v o l}=0.9-1.3$ across the whole range of collisionalities as seen in figure 6a. The data scatter could be attributed to the temporal evolution of the density profile for some discharges as shown in the analysis below.

Figure $6 \mathrm{~b}$ shows the analysis of particle fluxes for one of the discharges from the H-mode dataset. It is seen that the balance is dominated by the particle flux due to the neutral beam source $\Gamma_{\text {beam }}=\int s_{\text {beam }} d V / \int d S$ and the neoclassical Ware pinch $\Gamma_{W}=n_{e} V_{W}$ each equivalent to a normalised flux of $\left|\Gamma / n_{e}\right| \sim 0.5 \mathrm{~m} / \mathrm{s}$ at mid-radius. Wall neutrals contribute to the particle balance only towards the edge $r / a>0.6$ and their contribution is subject to large uncertainties due to poloidal inhomogeneities in diverted plasmas and the 1-D neutral particle model used in TRANSP. It is seen from figure $6 \mathrm{~b}$ that at mid radius the particle flux due to the neutral beam source and the Ware pinch are balanced by the rate of increase of particle content. As a result the diffusive particle flux is small:

$$
\Gamma_{\text {beam }}-n_{e} V_{W}-d / d t\left(\int n_{e} d V\right) / \int d S=-D_{e f f} \nabla n_{e} \sim 0 .
$$

Because of the flat density profile observed in these discharges the effective particle diffusivity $D_{\text {eff }}$ is undetermined. 
In future steady state devices such as a CTF [4] the Ware pinch will be zero. Density peaking can be achieved only by anomalous pinch or by core fuelling. In this CTF design at mid radius, $\Gamma_{\text {beam }} / n_{e}$ will be similar to values in the MAST plasma in figure $6 \mathrm{~b}$. Thus, subject to the value for particle diffusivity, the beam fuelling might contribute to the desired density peaking in a CTF device.

\section{Conclusions}

A dedicated experimental programme has been executed on the MAST tokamak with the aim of providing data for international confinement datasets. The data have been transmitted to the $\mathrm{H}$-mode confinement database and are improving the dataset used for development of ITER operating scenarios. The MAST data broadly support the recommended H-mode confinement formula in engineering parameters. In global dimensionless variables, MAST indicates similar favourable dependence on collisionality to that in conventional tokamaks. In addition, the data make a strong link between $\beta$ and aspect ratio dependence and thus show that the presently discussed interplay between $\beta$ and collisionality should be expanded to 3 variables including $\varepsilon$.

Energy confinement in L-mode exceeds the prediction of the current recommended confinement scaling suggesting that the MAST data would influence the aspect ratio dependence. The difference in confinement between L- and $\mathrm{H}$-mode plasmas with similar engineering parameters is mostly due to the lack of pedestal in L-mode.

In present H-modes in MAST, neo-classical pinch and neutral beam particle source are equal contributors to the particle balance in the core. Neutral beam fuelling will remain significant for a next step spherical tokamak such as a compact beam-driven CTF and thus could lead to desirable peaked density profiles. Density peaking will depend on the actual values of particle diffusivities and therefore future experiments in MAST should aim to quantify their values.

\section{Acknowledgement}

This work was funded by the United Kingdom Engineering and Physical Sciences Research Council and by EURATOM.

\section{References}

[1] Pankin, A. , McCune, D., Andre, R., et al., "The Tokamak Monte Carlo Fast Ion Module NUBEAM in the National Transport Code Collaboration Library", Computer Physics Communications 159 (2004) 157

[2] ITER Physics Basis, Nucl. Fusion 39 (1999) 2204

[3] Kardaun, O., Plasma Phys. and Contr. Fusion 41 (1999) 429

[4] Wilson, H., et al., this conference, FT/3-1Ra

[5] Petty, C. C., et al., Phys. Plasmas 6 (1999) 909

[6] Valovič, M., et al., 25 ${ }^{\text {th }}$ EPS Conf. ECA22C (1998) B122

[7] Thomsen, K., et al., $31^{\text {st }}$ EPS Conf. ECA28G (2004) P5.145

[8] Cordey, J. G., et al., this conference, IAEA-CN-116/IT/P3-32 